International Journal of Pure and Applied Mathematics

Volume 86 No. 3 2013, 487-500

ISSN: 1311-8080 (printed version); ISSN: 1314-3395 (on-line version)

url: http://www.ijpam.eu

doi: http://dx.doi.org/10.12732/ijpam.v86i3.3

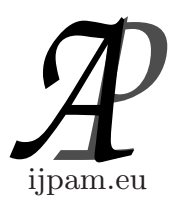

\title{
CONTROL DESIGN APPLIED TO A NON-IDEAL STRUCTURAL SYSTEM WITH BEHAVIOR CHAOTIC
}

\author{
Fábio Roberto Chavarette \\ Faculty of Engineering of Ilha Solteira \\ UNESP - Univ Estadual Paulista \\ Departament of Mathematics \\ Avenida Brasil, 56, 15385-000, Ilha Solteira, SP, BRAZIL
}

\begin{abstract}
In this paper we study the behavior of a structure vulnerable to excessive vibrations caused by an non-ideal power source. To perform this study, the mathematical model is proposed, derive the equations of motion for a simple plane frame excited by an unbalanced rotating machine with limited power (non-ideal motor). The non-linear and non-ideal dynamics in system is demonstrated with a chaotic behavior. We use a State-Dependent Riccati Equation Control technique for regulate the chaotic behavior, in order to obtain a periodic orbit small and to decrease its amplitude. The simulation results show the identification by State-Dependent Riccati Equation Control is very effective.
\end{abstract}

AMS Subject Classification: 34H10, 49N05

Key Words: state-dependent Riccati equation control, chaos control, nonideal system

\section{Introduction}

Most of the phenomena that occur have dynamic character, i.e., time-varying.

Received: February 28, 2013

(c) 2013 Academic Publications, Ltd. url: www.acadpubl.eu 
The representation of all these dynamic phenomena in the field of structural engineering is a big challenge. The great advancement of technology, large areas have been growing and improving, as the materials and construction techniques. What has made it possible to develop buildings, more slender and flexible.

In this paper, we approach the behavior of a structure vulnerable (linear dynamic model) the excessive excitation caused by a dynamic loading. This dynamic loading is a unbalanced rotating machine with limited power (nonideal motor), which characterizes the non-ideal system.

A system is said ideal when the excitation does not influence the response of the system, i.e., the ideal excitation has an ideal power source. On the other hand, when the excitation is influenced by the response of the system, the excitation is said to be non-ideal

The first characteristic of system non-ideal is that it presents an equation that describes the interaction of the energy source with the ideal dynamic system and the second characteristic, is observed that the dynamics of a non-ideal system approaches of the ideal case as the supplied potency becomes sufficiently big and vice-versa. Several contributions to the study of non-ideal problems were presented in the current literature, [1]-[5] and recently [6]-[9].

Among the various ways to minimize vibrations and reduce the oscillatory motion caused by non-ideal power source in the structure is discussed [13] in this paper the application of the structural control.

One technique that has shown considerable promitent in simulations involves the approximate solutions of a nonlinear, state-dependent Riccati equation for the dynamical system in discrete time steps to calculate a feedback control law that is optimized around the system state-tracking control, in which the cost function to be minimized is quadratic in the difference between the actual or estimated state and a commanded state trajectory. This technique is called State-Dependent Riccati Equation (SDRE) control [10, 11] techniques can be applied to solve a wide range of problems [10, 12].

The aim of this paper is to propose the application of the State-Dependent Riccati Equation Control [18] to control the chaotic movement of a non-ideal structural system.

We organize the paper as it follows: in Section 2, we demonstrated the mathematical model and we showed of the non-ideal dynamics of the model. In Section 3, we discussed a control design problem for non-ideal model. In Section 4, we made the concluding remarks of this paper. Finally, we listed out the bibliographic references which we used in this paper. 


\section{Non-Ideal Structural System}

An introduction to mathematical problem that is proposed, derive the equations of motion for a simple plane frame under excitation in vertical direction, as shown in Figure 1. where $x_{1}=a+S+q_{1}$ and $x_{2}=b+S+q_{2}$, where $S$ is the

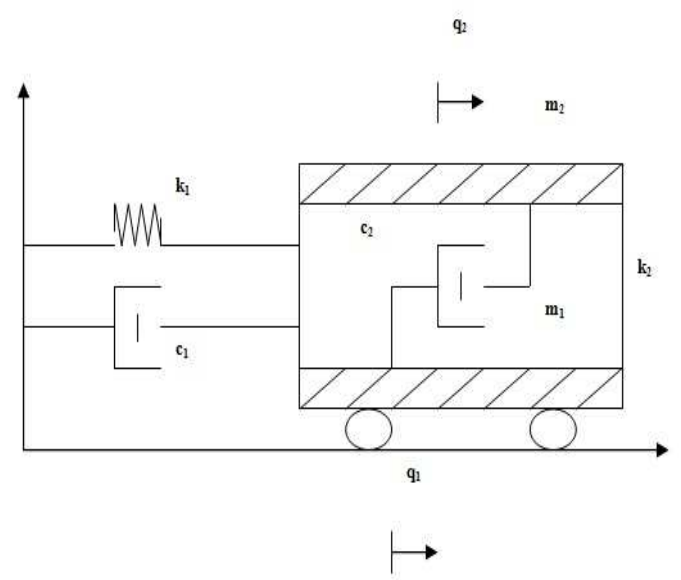

Figure 1: Structural System

non-ideal excitation.

The total kinetic energy $(\mathrm{T})$ of the system is:

$$
T=1 / 2\left[m_{1}\left(\dot{S}+\dot{q}_{1}\right)^{2}+m_{2}\left(\dot{S}+\dot{q}_{2}\right)^{2}\right]
$$

The total potential energy $(\mathrm{V})$ of the system is:

$$
V=1 / 2\left[k_{1} q_{1}^{2}+k_{2}\left(q_{2}-q_{1}\right)^{2}\right]
$$

The Lagrangian $(\mathrm{L}=\mathrm{T}-\mathrm{V})$ is:

$$
L=1 / 2\left[m_{1}\left(\dot{S}+\dot{q}_{1}\right)^{2}+m_{2}\left(\dot{S}+\dot{q}_{2}\right)^{2}-k_{1} q_{1}^{2}-k_{2}\left(q_{2}-q_{1}\right)^{2}\right]
$$

The Lagrange equation for the generalized coordinate $q_{1}$ is:

$$
m_{1}\left(\ddot{q}_{1}+\ddot{S}\right)+k_{1} q_{1}-k_{2}\left(q_{2}-q_{1}\right)=-c_{1} \dot{q}_{1}
$$

The Lagrange equation for the generalized coordinate $q_{2}$ is:

$$
m_{2}\left(\ddot{q}_{2}+\ddot{S}\right)+k_{2}\left(q_{2}-q_{1}\right)+c_{2}\left(q_{2}-q_{1}\right)=-c_{1} \dot{q}_{1}
$$


Thus, the system can be modeled by the equations:

$$
\begin{aligned}
m_{1} \ddot{q}_{1}+k_{1} q_{1}-k_{2}\left(q_{2}-q_{1}\right)+c_{1} \dot{q}_{1} & =m_{1} \ddot{S} \\
m_{2} \ddot{q}_{2}+k_{2}\left(q_{2}-q_{1}\right)+c_{2}\left(\dot{q}_{2}-\dot{q}_{1}\right) & =m_{2} \ddot{S} \\
\ddot{q}_{1}+\frac{k_{1}}{m_{1}} q_{1}-\frac{k_{2}}{m_{1}}\left(q_{2}-q_{1}\right)+\frac{c_{1}}{m_{1}} \dot{q}_{1} & =-\ddot{S} \\
\ddot{q}_{2}+\frac{k_{2}}{m_{2}}\left(q_{2}-q_{1}\right)+\frac{c_{2}}{m_{2}}\left(\dot{q}_{2}-\dot{q}_{1}\right) & =-\ddot{S}
\end{aligned}
$$

being $\omega_{1}^{2}=\frac{k_{1}+k_{2}}{m_{1}} ; \omega_{2}^{2}=\frac{k_{2}}{m_{2}}$ has

$$
\begin{aligned}
\ddot{q}_{1}+\omega_{1}^{2} q_{1}-\frac{k_{2}}{m_{1}} q_{2}+\frac{c_{1}}{m_{1}} \dot{q}_{1} & =-\ddot{S} \\
\ddot{q}_{2}+\omega_{2}^{2}\left(q_{2}-q_{1}\right)+\frac{c_{2}}{m_{2}}\left(\dot{q}_{2}-\dot{q}_{1}\right) & =-\ddot{S}
\end{aligned}
$$

where $S$ is the non-ideal excitation (unbalanced rotating machine with limited power)

$$
\begin{array}{r}
\ddot{z}+b \dot{z}=r \ddot{q}_{1} \sin z+a \\
S=p\left(\dot{z}^{2} \cos z+\ddot{z} \sin z\right)
\end{array}
$$

Where $z$ is non-ideal excitation response, $a, b, p$ and $r$ are dimensionless constant positives. The term $S$ is due interaction between the dynamical system and an energy source, for example, a DC motor with limited power supply. $S$ is responsible to the Non-idealization of the system. Note also that in the equation (12) we may observe an interaction term . The parameter $a$ is a constant depending on initial conditions and $b$ is an internal damping in Dc Motor.

Making $x_{1}=q_{1}, x_{2}=\dot{q}_{1}, x_{3}=q_{2}, x_{4}=\dot{q}_{2}, x_{5}=z$ and $x_{6}=\dot{z}$, has

$$
\begin{array}{r}
\dot{x}_{1}=x_{2} \\
\dot{x}_{2}=-\omega_{1}^{2} x_{1}+\frac{k_{2}}{m_{1}} x_{3}-\frac{c_{1}}{m_{1}} x_{2}-\left(p\left(x_{6}^{2} \cos x_{5}+x_{6} \sin x_{5}\right)\right) \\
\dot{x}_{3}=x_{4} \\
\dot{x}_{4}=\omega_{2}^{2}\left(x_{1}-x_{3}\right)+\frac{c_{2}}{m_{2}}\left(x_{2}-x_{4}\right)-\left(p\left(x_{6}^{2} \cos x_{5}+x_{6} \sin x_{5}\right)\right) \\
\dot{x}_{5}=x_{6} \\
\dot{x}_{6}=-b x_{6}^{2}+r x_{2} \sin x_{5}+a
\end{array}
$$


(a)

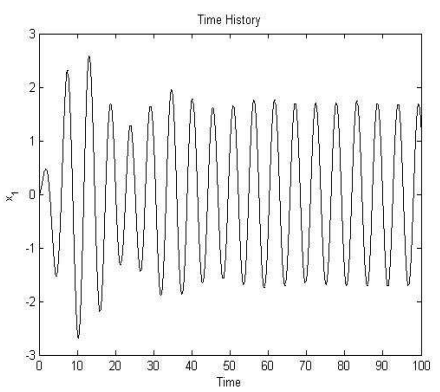

(c)
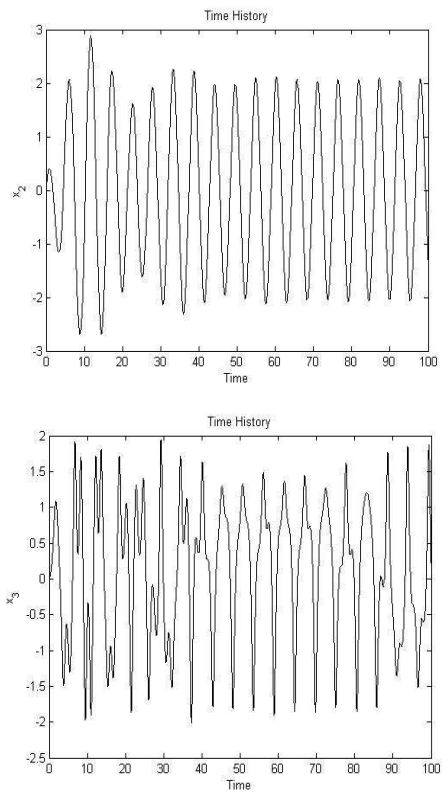

(e)

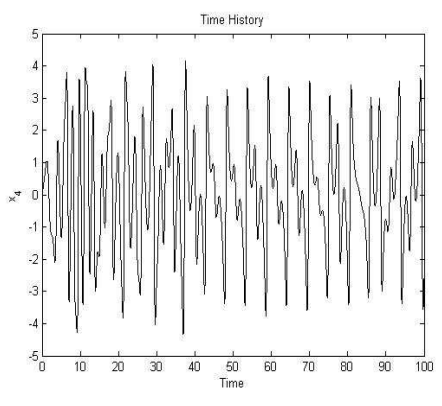

(b)

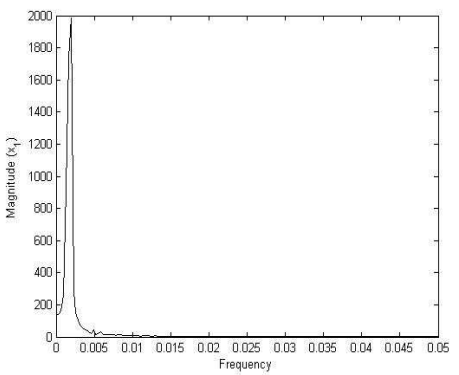

(d)

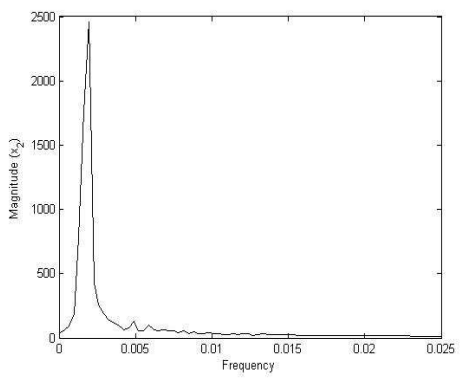

(f)

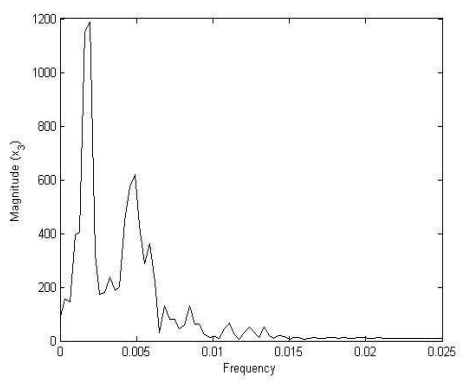

(h)

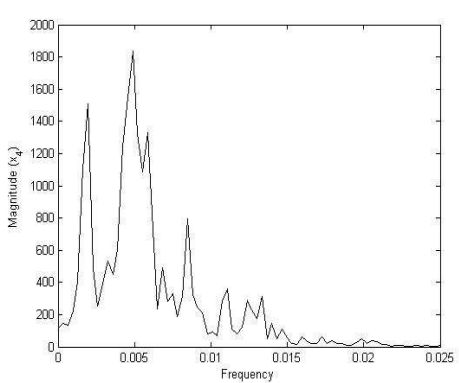

Figure 2: (a)Dynamical behavior of the time history $\left(x_{1}\right)$, (b) FFT for $\left(x_{1}\right)$, (c) Time history $\left(x_{2}\right)$, (d) FFT $\left(x_{2}\right)$, (e) Time history $\left(x_{3}\right)$, (f) FFT $\left(x_{3}\right),(\mathrm{g})$ Time history $\left(x_{4}\right)$, and (h) FFT $\left(x_{4}\right)$. 
The Figure 2 illustrate the dynamics behavior of the adopted dynamics model, by using numerical values, for the chosen parameters $\omega_{1}=1, m_{1}=7$, $c_{1}=0.6, k_{2}=0.8, \omega_{2}=2, c_{2}=0.7, m_{2}=7$ to the structural system and $a=2.3, b=2.0, r=0.5$ and $p=0.5$ to the non-ideal excitation.

The Figure 3 show the portrait phase for the chaotic behavior.

(a)

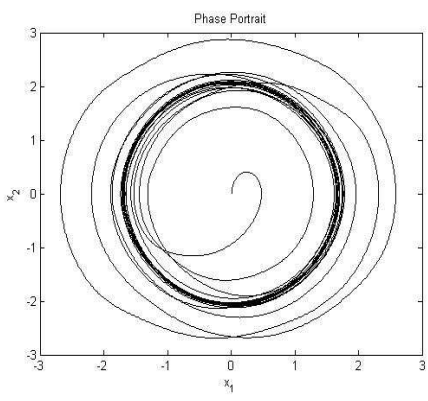

(b)

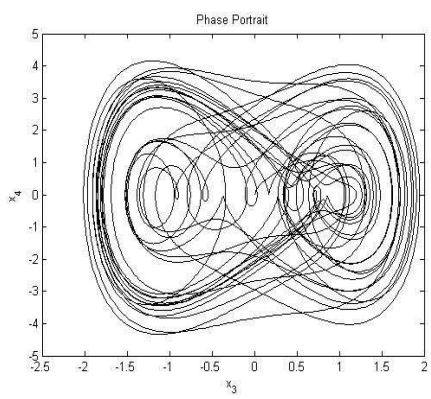

Figure 3: Phase Portrait. (a) $x_{1}$ and $x_{2}$ projections and (b) $x_{3}$ and $x_{4}$ projections

Figure 4 illustrates the four Lyapunov exponents, $\lambda_{1}=0.077975 ; \lambda_{2}=$ $-0.039748 ; \lambda_{3}=-0.047754 ; \lambda_{4}=-0.052616 ; \lambda_{5}=-0.0393-4$ and $\lambda_{6}=$ -0.11427 , demonstrating the presence of the chaos with the one Lyapunov exponent positive. The total time for Lyapunov exponents computation was the $\Delta \tau=100,000$ with time-step of $\tau=0.5$.

(a)

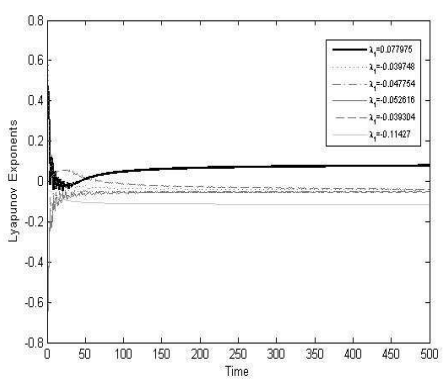

(b)

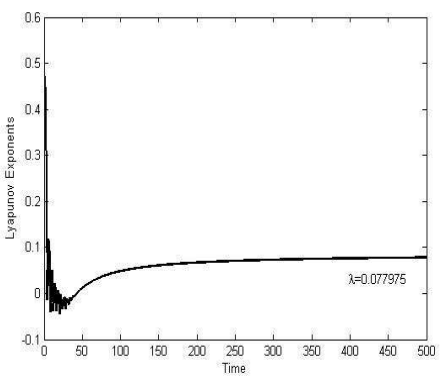

Figure 4: Dynamics of Lyapunov expoents for system (a) Exponents (b) Positive Exponent

Aiming to minimize vibrations and reduce the oscillatory motion caused in the system in the following section proposes the application of State-Dependent Riccati Equation (SDRE) to reduce this chaotic motion, see Figure 4, to a small 
stable orbit.

\section{Control Design}

An alternative to minimize vibrations unstable presented is the structural control. The structural control, basically promotes changes in stiffness and damping of the structure, either by adding external devices, either by the action of external forces. We can adopt various control models, such as the passive control, active control, hybrid control, and semi-active control. In this work we adopt the State-Dependent Riccati Equation Control proposed by Pearson (1962) and later expanded by Wernli and Cook [14], was independently studied by Mracek and Cloutier [15] and alluded to by Friedland [16]. Use of the State-Dependent Riccati Equation (SDRE) to control nonlinear system has seen increasing application [17].

$$
J=\frac{1}{2} \int_{t_{0}}^{\infty} x^{t} Q(x) x+u^{T} R(x) u d t
$$

with respect to the state $x$ and control $u$ subject to the nonlinear differential constraint

$$
\dot{x}=f(x)+g(x) u
$$

where $x \epsilon R^{n}, y \epsilon R^{n}, f(x) \epsilon C^{k}, g(x) \epsilon C^{k}, Q(x) \epsilon C^{k}, R(x) \epsilon C^{k}, k \geq 1$ and where $Q(x)=$ $C^{T}(x) C(x) \geq 0$, and $R(x)>0$ for all $x$. Here it is assumed that $f(0)=0$ and $g(x) \neq 0$ for all $x$. It may also be desirable to select $Q(x)$ and $R(x)$ such that the performance index $J(x, u)$ in $(20)$ is globally convex. We seek stabilizing approximate solutions of problem (20)-(21) of the form $u=\phi(x)$ where $\phi$ is a nonlinear function of $x$.

\subsection{State-Dependent Coefficient Form}

It is well-know that the nonlinear dynamics (22) can be represented by the following linear structure having state-dependent coefficients:

$$
\dot{x}=A(x) x+B(x) u
$$

where

$$
f(x)=A(x) x
$$




$$
B(x)=g(x)
$$

It is also know [7] that, in the multivariable case, there are an infinite number of ways to bring the nonlinear system to SDC form. Associated with the SDC form, we have the following definitions.

$A(x)$ is an observable (detectable) parameterization of the nonlinear system (in region $\Omega$ ) if the par $\{C(x), A(x)\}$, is pointwise observable (detectable) in the linear sense for all $x[\epsilon \Omega]$.

$A(x)$ is a controllable (stabilizable) parameterization of the nonlinear system (in a region $\Omega$ ) if the pair $\{B(x), A(x)\}$ is pointwise controllable (stabilizable) in the linear sense for all $x[\epsilon \Omega]$.

\subsection{Application of the Control Design Theory to a Non-Idel Model}

The non-linear state model (21) can written in a linear state dependent coefficient (SDC) form of (24), where the vector $x=\left[x_{1} x_{2} x_{3} x_{4} x_{5} x_{6}\right]^{T}$ represents the system states the time dependent, $\dot{x} \epsilon \Re^{2}$ is the vector of first derivatives of the state, $\dot{x} \epsilon \Re^{1}$ is the control function. $U$ is the force applied to control. Considering the initial and end conditions as: $x\left(t_{0}\right)=x_{0}, x(\infty)=0$ The state dependent coefficients are given by:

$$
\begin{gathered}
A=\left[\begin{array}{cccccc}
0 & 1 & 0 & 0 & 0 & 0 \\
-1 & -0.0857 & 0.1142 & 0 & 0 & 0 \\
0 & 0 & 0 & 1 & 0 & 0 \\
4 & 0.1 & -4 & -0.1 & 0 & 0 \\
0 & 0 & 0 & 0 & 0 & 1 \\
0 & 0.4207 & 0 & 0 & 0.0027 & -4
\end{array}\right] \\
B(x)=\left[\begin{array}{c}
1 \\
1 \\
1 \\
1 \\
1 \\
1
\end{array}\right] \\
\quad Q(x)=S^{T}(x) S(x) \\
\quad \operatorname{diag}\left\{\sqrt{q_{i}}\right\}_{i=1, \ldots 6}
\end{gathered}
$$


Rewritten (22),

$$
\dot{x}=A(x), x+\left(\begin{array}{l}
1 \\
1 \\
1 \\
1 \\
1 \\
1
\end{array}\right) u
$$

being $x$ a vector.

The state $x$ and $u$ the control are given for function $f(x)=A(x), b(x)=$ $B(x)$ e $d(x)=S(x) x[18]$.

Considering the functional to be minimized is

$$
J=\frac{1}{2} \int_{t_{0}}^{\infty} x^{t} Q(x) x+u^{T} R(x) u d t
$$

where

$$
Q=\left[\begin{array}{llllll}
1 & 0 & 0 & 0 & 0 & 0 \\
0 & 1 & 0 & 0 & 0 & 0 \\
0 & 0 & 1 & 0 & 0 & 0 \\
0 & 0 & 0 & 1 & 0 & 0 \\
0 & 0 & 0 & 0 & 1 & 0 \\
0 & 0 & 0 & 0 & 0 & 1
\end{array}\right]
$$

and $\mathrm{R}=[1]$.

Construct the nonlinear feedback controller via

$$
u=-R^{-1}(x) B^{T}(x) P(x) x
$$

Solve the state-dependent Riccati equation (SDRE)

$$
A^{T}(x) P+P A(x)-P B(x) R^{-1}(x) B^{T}(x) P+Q(x)=0
$$

Mracek and Cloutier [15], in the region $\Omega$ about the origin of the SDRE method provides a solution in an asymptotically stable limit cycle. In the case of a scalar solution, the method achieves an optimal solution to functional (28), even when $Q$ and $R$ are functions of $x$.

The SDRE control meet the first and second necessary condition of optimism, $H_{u}=0(H$ is the Hamiltonian of the problem (24)-(28)). The Hamiltonian for the control is:

$$
H(x, u, \lambda)=\frac{1}{2}\left(x^{T} Q x+u^{T} R u\right)+\lambda^{T}(A(x) x+B(x) u)
$$


The conditions necessary for the control are [18]:

$$
\begin{array}{r}
\dot{\lambda}=-Q x-\frac{1}{2}\left[\frac{\partial A(x) x}{\partial x}\right]^{T} \lambda-\left[\frac{\partial(B(x) u)}{\partial x}\right]^{T} \lambda, \\
\dot{x}=A(x) x+B(x) u, \\
0=R u+B(x) \lambda .
\end{array}
$$

Here

$$
u=-R^{-1}(x) B^{T}(x) \lambda
$$

Assuming a co-state, it is known that:

$$
\lambda=P(x) x
$$

Substituting (33) in (34) are obtained by SDRE control (29).

The trajectories of the system with control may be seen, through Figures $5-14$.

\section{Conclusions}

In this work, a dynamics of the Non-ideal Structural System with behavior chaotic is proposed and investigated.

We proposed the use of an SDRE control strategy to reduce the chaotic movement of this system to a small stable orbit.

The Figures 5 and 6 illustrated the effectiveness of the control strategy taken to these problems. 
(a)

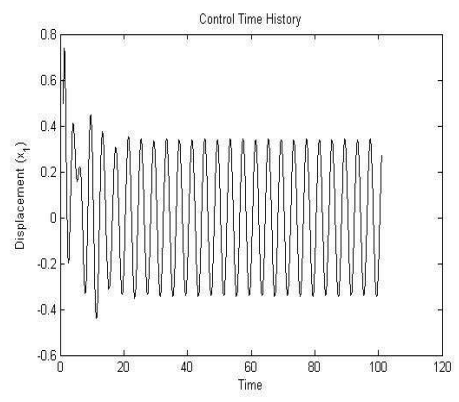

(c)

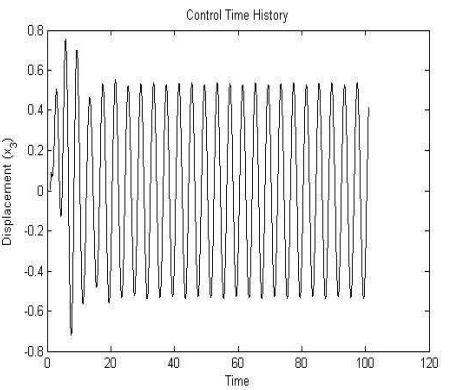

(e)

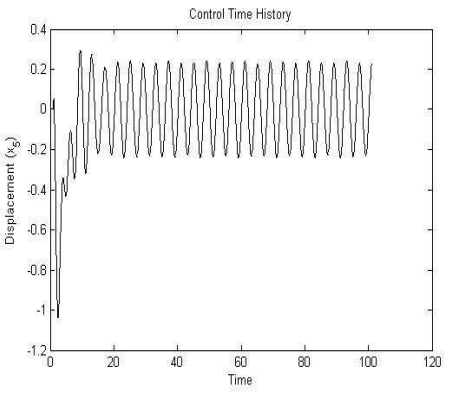

(b)

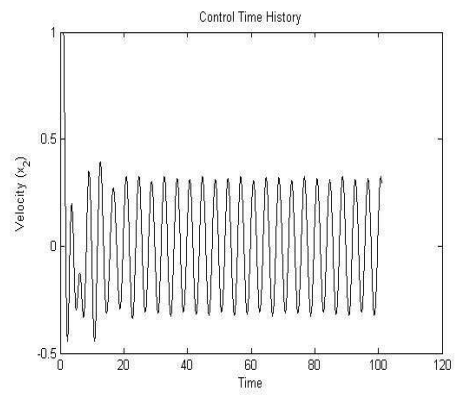

(d)
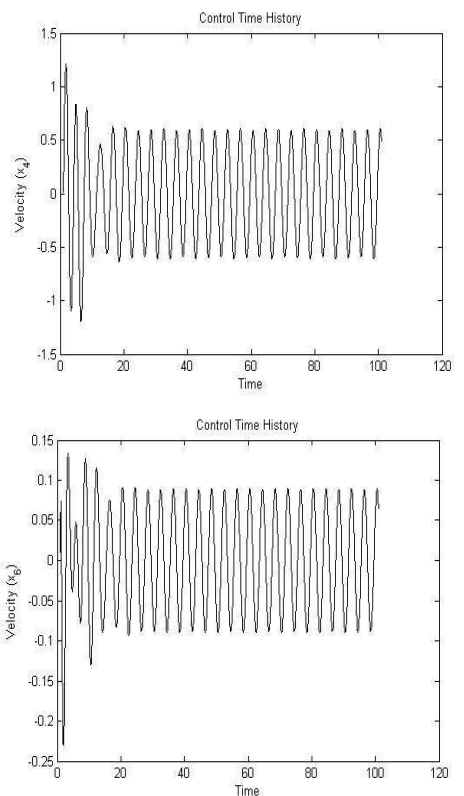

Figure 5: Dynamical behavior of the time history (a) $x_{1}$, (b) $x_{2}$, (c) $x_{3}$, (d) $x_{4}$, (e) $x_{5}$ and (f) $x_{6}$. 
(a)

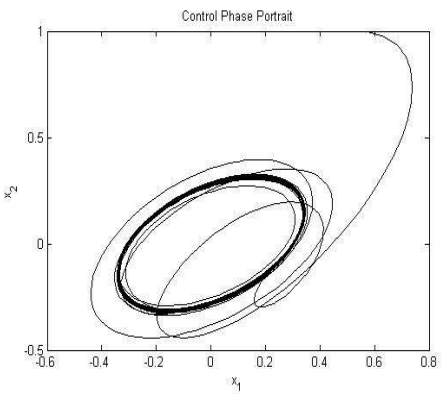

(b)
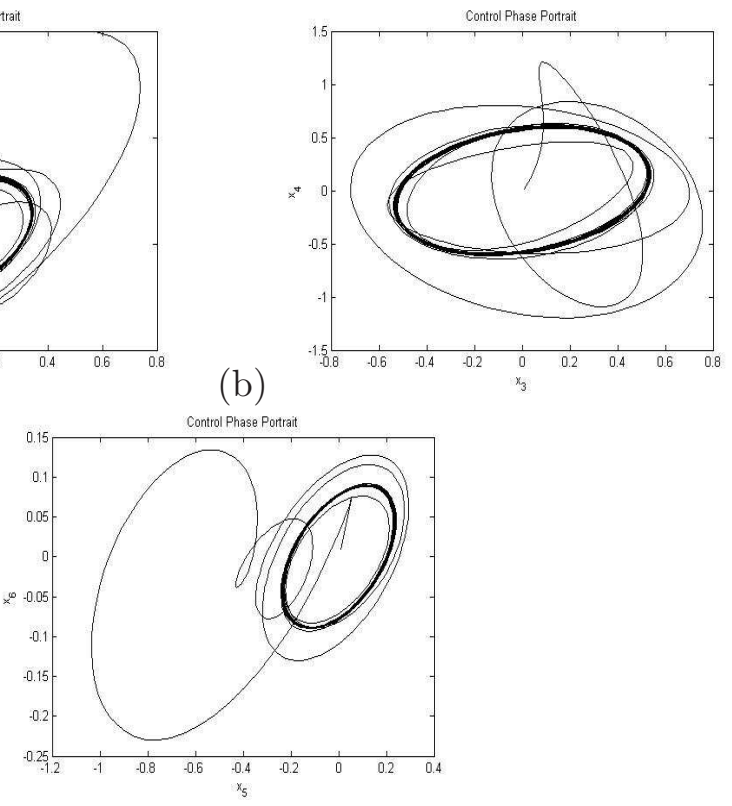

(c)

Figure 6: Control Phase Portrait. (a) $x_{1}$ and $x_{2}$ projections, (b) $x_{3}$ and $x_{4}$ projections and (c) $x_{5}$ and $x_{6}$ projections.

\section{Acknowledgments}

The author thanks Conselho Nacional de Pesquisas (CNPq) for a financial supports (Proc.n. 301769/2012-5).

\section{References}

[1] I.I. Blekman, Self-Synchronization of certain vibratory devices, Engineering Transactions, 16, ASME PRESS Translations, Wayne State University, USA (1953).

[2] R.M. Evan-Iwanowski, Resonance Oscillators in Mechanical Systems, J. Appl. Mech, 44 (1977), 365-367, doi: 10.1115/1.3424080.

[3] M.F. Dimentberg, Statistical Dynamics of Nonlinear and Time Varying Systems, John Wiley and Sons (1988).

[4] Dimentberg, M.F., McGovern, L., Norton, R.L., Chapdelaine, J. e Harrison, R., Dynamics of an Unbalanced Shaft Interacting with a Lim- 
ited Power Supply, Non-linear Dynamics, 13 (1997), 171-187, doi: 10.1023/A:1008205012232.

[5] A.H. Nayfeh, D.T. Mook, Non-linear Oscillations, John Wiley e Sons, New York (1979).

[6] F.R. Chavarette, J.M. Balthazar, J.L.P. Felix, M. Rafikov, M, A reducing of a chaotic movement to a periodic orbit, of a micro-electro-mechanical system, by using an optimal linear control design, Communications in Nonlinear Science and Numerical Simulation, 14, No. 5 (2009), 1844-1853, doi: 10.1016/j.cnsns.2008.09.003.

[7] F.R. Chavarette, J.M. Balthazar, J.L.P. Felix, Remarks on an Optimal Linear Control Design Applied to a Nonideal and an Ideal Structure Coupled to an Essentially Nonlinear Oscillator, J. Comput. Nonlinear Dynam., 5, No. 2 (2010), 024501-024508, 2010, doi: 10.1115/1.4000829.

[8] F.R. Chavarette, J.M. Balthazar, M. Rafikov, H.A. Hermini, On non-linear dynamics and an optimal control synthesis of the action potential of membranes (ideal and non-ideal cases) of the Hodgkin Huxley (HH) mathematical model. Chaos, Solitons and Fractals, 39 (2009), 1651-1666, doi: 10.1016/j.chaos.2007.06.016.

[9] F.R. Chavarette, J.M. Balthazar, N.J. Peruzzi, M. Rafikov, On non-linear dynamics and control designs applied to the ideal and non-ideal variants of the Fitzhugh-Nagumo (FN) mathematical model, Communications in Nonlinear Science and Numerical Simulation, 14, No. 3 (2009), 892-905, doi: 10.1016/j.cnsns.2007.10.016.

[10] J. R. Coultier, C.N. Souza, C.P. Mracek, Nonlinear regulation and nonlinear H-infinity control via the state-dependent Riccati equation technique; part 1, theory; part 2, examples. In: Proceedings of the International Conference on Nonlinear Problems in Aviation and Aerospace. Available through University Press, Embry-Riddle Aeronautical University, Daytona Beach (1996), 117-141.

[11] C.P. Mracek, J.R. Cloutier, C.N. D'Souza, A new technique for nonlinear estimation. In: Proceedings of the IEEE Conference on Control Applications, Dearborn, MI (1996), 338-343.

[12] A. Fenili, J.M. Balthazar, The rigid-flexible nonlinear robotic manipulator: modeling and control, Communications in Nonlinear Science and 
Numerical Simulation, 16, No.5 (2011), 2332-2341, doi: 10.1016/j.cnsns. 2010.04.057.

[13] A.M. Tusset, J.M. Balthazar, F.R. Chavarette, J.L.P. Felix, On Energy transfer phenomena, in a nonlinear ideal and non-ideal essential vibrating systems, coupled to a (MR) Magneto-rheological damper. Nonlinear Dynamics, 69 (2012), 1859-1880, doi: 10.1007/s11071-012-0391-5.

[14] A. Wernli, G. Cook, Suboptimal control for the nonlinear control design techniques on a model of the Caltech ducted fan, Automatica, 37 (2001), 1971-1978.

[15] C.P. Mracek, J.R. Cloutier, Control designs for the nonlinear benckmark problem via the state-dependent Riccati equation method. International Journal of Robust and Nonlinear Control, 8 (1998), 401-433.

[16] B. Friedland, Advanced Control System Design, Prentice-Hall, Englewood Cliffs, NJ (1996).

[17] A.M. Shawky, A.W. Ordys, L. Petropoulakis, M.J. Grimble, Position control of flexible manipulator using non-linear with state-dependent Riccati equation, In: Proceedings IMechE, Part I: J. Systems and Control Engineering, 221 (2007), 475-486.

[18] H. T. Banks, B.M. Lewis, H.T. Tran, Nonlinear feedback controllers and compensators: a state-dependent Riccati equation approach. Comp. Optim. Appl., 37 (2007), 177-218, doi: 10.1007/s10589-007-9015-2. 